\title{
Mining Idioms from Source Code
}

\author{
Miltiadis Allamanis, Charles Sutton \\ School of Informatics, University of Edinburgh \\ Edinburgh EH8 9AB, UK \\ \{m.allamanis,csutton\}@ed.ac.uk
}

\begin{abstract}
We present the first method for automatically mining code idioms from a corpus of previously written, idiomatic software projects. We take the view that a code idiom is a syntactic fragment that recurs across projects and has a single semantic purpose. Idioms may have metavariables, such as the body of a for loop. Modern IDEs commonly provide facilities for manually defining idioms and inserting them on demand, but this does not help programmers to write idiomatic code in languages or using libraries with which they are unfamiliar. We present HAGGIs, a system for mining code idioms that builds on recent advanced techniques from statistical natural language processing, namely, nonparametric Bayesian probabilistic tree substitution grammars. We apply HAGGIs to several of the most popular open source projects from GitHub. We present a wide range of evidence that the resulting idioms are semantically meaningful, demonstrating that they do indeed recur across software projects and that they occur more frequently in illustrative code examples collected from a Q\&A site. Manual examination of the most common idioms indicate that they describe important program concepts, including object creation, exception handling, and resource management.
\end{abstract}

Categories and Subject Descriptors: D.2.3 [Software Engineering]:Coding Tools and Techniques

General Terms: Documentation, Languages, Algorithms

Keywords: syntactic code patterns, code idioms, naturalness of source code

\section{INTRODUCTION}

Programming language text is a means of human communication. Programmers write code not simply to be executed by a computer, but also to communicate the precise details of the code's operation to later developers who will adapt, update, test and maintain the code. It is perhaps for this reason that source code is natural in the sense described by Hindle et al. [18]. Programmers themselves use the term idiomatic to refer to code that is written in a manner that other experienced developers find natural. Programmers believe that it is important to write idiomatic code, as evidenced by the amount of relevant resources available: For example, Wikibooks has a book devoted to $\mathrm{C}++$ idioms [52], and similar guides are available for Java [22] and JavaScript [9, 50]. A guide on GitHub for idiomatic JavaScript [50] has more 6,644 stars and 877 forks. A search for the keyword "idiomatic" on Stack Overflow yields over 49,000 hits; all but one of the first 100 hits are questions about what the idiomatic

Permission to make digital or hard copies of all or part of this work for personal or classroom use is granted without fee provided that copies are not made or distributed for profit or commercial advantage and that copies bear this notice and the full citation on the first page. To copy otherwise, to republish, to post on servers or to redistribute to lists, requires prior specific permission and/or a fee.

FSE' 14 Hong Kong, China

Copyright 2014 ACM /14/11 ...\$15.00. method is for performing a given task.

The notion of code idiom is one that is commonly used but seldom defined. We take the view that an idiom is a syntactic fragment that recurs frequently across software projects and has a single semantic purpose. Idioms may have metavariables that abstract over identifier names and code blocks. For example, in Java the loop for (int $i=0 ; i<n ; i++)\{\ldots\}$ is a common idiom for iterating over an array. It is possible to express this operation in many other ways, such as a do-while loop or using recursion, but as experienced Java programmers ourselves, we would find those alternatives alien and more difficult to understand. Idioms differ significantly from previous notions of textual patterns in software, such as code clones [44] and API patterns [56]. Unlike clones, idioms commonly recur across projects, even ones from different domains, and unlike API patterns, idioms commonly involve syntactic constructs, such as iteration and exception handling. A large number of example idioms, all of which are automatically identified by our system, are shown in Figures 6 and 7.

Major IDEs currently support idioms by including features that allow programmers to define idioms and easily reuse them. Eclipse's SnipMatch [43] and IntelliJ IDEA's live templates [23] allow the user to define custom snippets of code that can be inserted on demand. NetBeans includes a similar "Code Templates" feature in its editor. Recently, Microsoft created Bing Code Search [36] that allows users to search and add snippets to their code, by retrieving code from popular coding websites, such as Stack Overflow. The fact that all major IDEs include features that allow programmers to manually define and use idioms attests to their importance.

We are unaware, however, of methods for automatically identifying code idioms. This is a major gap in tooling for software development. Software developers cannot use manual IDE tools for idioms without significant effort to organize the idioms of interest and then manually add them to the tool. This is especially an obstacle for less experienced programmers who do not know which idioms they should be using. Indeed, as we demonstrate later, many idioms are library-specific, so even an experienced programmer will not be familiar with the code idioms for a library that is new to them. Although in theory this cost could be amortized if the users of each library were to manually create an idiom guide, in practice even expert developers will have difficulty exhaustively listing all of the idioms that they use daily, just as a native speaker of English would have difficulty exhaustively listing all of the words that they know. Perhaps for this reason, although IDEs have included features for manually specifying idioms for many years, ${ }^{1}$ we are unaware of large-scale efforts by developers to list and categorize library-specific idioms. The ability to automatically identify idioms is needed.

In this paper, we present the first method for automatically mining code idioms from an existing corpus of idiomatic code. At first, this might seem to be a simple proposition: simply search for subtrees that occur often in a syntactically parsed corpus. How-

\footnotetext{
${ }^{1}$ See, e.g., http://bit.ly/1nN $4 h z 6$
} 
ever, this naive method does not work well, for the simple reason that frequent trees are not necessarily interesting trees. To return to our previous example, for loops occur more commonly than for (int $i=\mathbb{Q} ; i<n ; i++)\{\ldots\}$, but one would be hard pressed to argue that for $(\ldots)\{\ldots\}$ on its own (that is, with no expressions or body) is an interesting pattern.

Instead, we rely on a different principle: interesting patterns are those that help to explain the code that programmers write. As a measure of "explanation quality", we use a probabilistic model of the source code, and retain those idioms that make the training corpus more likely under the model. These ideas can be formalized in a single, theoretically principled framework using a nonparametric Bayesian analysis. Nonparametric Bayesian methods have become enormously popular in statistics, machine learning, and natural language processing because they provide a flexible and principled way of automatically inducing a "sweet spot" of model complexity based on the amount of data that is available [41, 16, 48]. In particular, we employ a nonparametric Bayesian tree substitution grammar, which has recently been developed for natural language [10, 42], but which has not been applied to source code.

Because our method is primarily statistical in nature, it is language agnostic, and can be applied to any programming language for which one can collect a corpus of previously-written idiomatic code. Our major contributions are:

- We introduce the idiom mining problem (Section 2);

- We present HagGis, a method for automatically mining code idioms based on nonparametric Bayesian tree substitution grammars (Section 3);

- We demonstrate that HaGGIs successfully identifies cross-project idioms (Section 5), for example, $67 \%$ of idioms that we identify from one set of open source projects also appear in an independent set of snippets of example code from the popular Q\&A site Stack Overflow;

- Examining the idioms that HaGgis identifies (Figure 6), we find that they describe important program concepts, including object creation, exception handling, and resource management;

- To further demonstrate that the idioms identified by HaGGIS are semantically meaningful, we first examine the relationship between idioms and code libraries (Section 5.4), finding that many idioms are strongly connected to package imports in a way that can support suggestion.

We submitted a small set of idioms from HaGGIS to the Eclipse Snipmatch project (Section 5.3) for inclusion into its presupplied library of snippets. Several of these snippets have already been accepted.

\section{PROBLEM DEFINITION}

A code idiom is a syntactic fragment that recurs across software projects and serves a single semantic purpose. An example of an idiom is shown in Figure 1(b). This is an idiom which is used for manipulating objects of type android. database.Cursor, which ensures that the cursor is closed after use. (This idiom is indeed discovered by our method.) As in this example, typically idioms have parameters, which we will call metavariables, such as the name of the Cursor variable, and a code block describing what should be done if the moveToFirst operation is successful. An Android programmer who is unfamiliar with this idiom might make bad mistakes, like not calling the close method or not using a finally block, causing subtle memory leaks.

Many idioms, like the close example or those in Figure 6, are specific to particular software libraries. Other idioms are general across projects of the same programming language, such as those in Figure 7, including an idiom for looping over an array or an idiom defining a String constant. (All of the idioms in these figures are discovered by our method.) Idioms concerning exception handling and resource management are especially important because they help to ensure correctness properties. As these examples show, idioms are usually parametrized and the parameters often have syntactic structure, such as expressions and code blocks.

We define idioms formally as fragments of abstract syntax trees, which allows us to naturally represent the syntactic structure of an idiom. More formally, an idiom is a fragment $\mathcal{T}=(V, E)$ of an abstract syntax tree (AST). By fragment, we mean the following. Let $G$ be the context-free grammar ${ }^{2}$ of the programming language in question. Then a fragment $\mathcal{T}$ is a tree of terminals and nonterminal from $G$ that is a subgraph of some valid parse tree from $G$.

An idiom $\mathcal{T}$ can have as leaves both terminals and non-terminals. Non-terminals correspond to metavariables which must be filled in when instantiating the idiom. For example, in Figure 1(c), the shaded lines represent the fragment for an example idiom; notice how the Block node of the AST, which is a non-terminal, corresponds to a \$BODY\$ metavariable in the pattern.

Idiom mining Current IDEs provide tools for manually defining idioms and inserting them when required, but this requires that the developer incur the required setup cost, and that the developer know the idioms in the first place. To eliminate these difficulties, we introduce the idiom mining problem, namely, to identify a set of idioms automatically given only a corpus of previously-written idiomatic code. More formally, given a training set of source files with abstract syntax trees $\mathcal{D}=\left\{T_{1}, T_{2}, \ldots T_{N}\right\}$, the idiom mining problem is to identify a set of idioms $\mathcal{I}=\left\{\mathcal{T}_{i}\right\}$ that occur in the training set. This is an unsupervised learning problem, as we do not assume that we are provided with any example idioms that are explicitly identified. Each fragment $\mathcal{T}_{i}$ should occur as a subgraph of every tree in some subset $\mathcal{D}\left(\mathcal{T}_{i}\right) \subseteq \mathcal{D}$ of the training corpus.

What Idioms are Not Idioms are not clones. Code clones [44, 45, $5,6,24,29$ ] are pieces of code that are used verbatim (or nearly so) in different code locations due to copy paste operations. By contrast, idioms are used verbatim (or nearly so) in different code locations because programmers find them natural for performing a particular task. Essentially, idioms have a semantic purpose that developers are consciously aware of. Indeed, unlike clones we suggest that idioms are not typically entered by copy-paste - speaking for ourselves, we do not need copy-paste to enter something as simple as for (int $i=0 ; i<n ; i++)$. Rather, we suggest that programmers treat idioms as mental chunks, which they often type directly by hand when needed, although we leave this conjecture to future work.

Because methods for clone detection work by finding repeated regions of code, existing clone detection methods could also be applied to find idioms. However, in our experiments (Section 5.2), this does not prove to be an effective approach. We argue that this highlights a conceptual difference between clone detection and idiom detection: Clone detection methods attempt to find the largest fragment that is copied, whereas methods for idiom detection need to search for fragments that seem "natural" to programmers, which requires a trade off between the size of the fragment and the frequency with which programmers use it.

Also, idiom mining is not API mining. API mining $[38,51,56]$ is an active research area that focuses on mining groups of library functions from the same API that are commonly used together. These

${ }^{2}$ Programming language grammars typically describe parse trees rather than ASTs, but since there is a 1:1 mapping between the two, we assume a CFG that directly describes ASTs is available. 


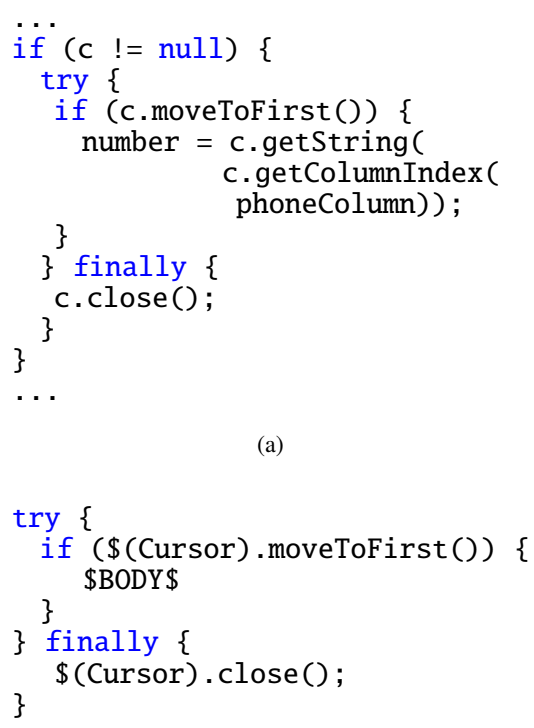

(a)

(b)

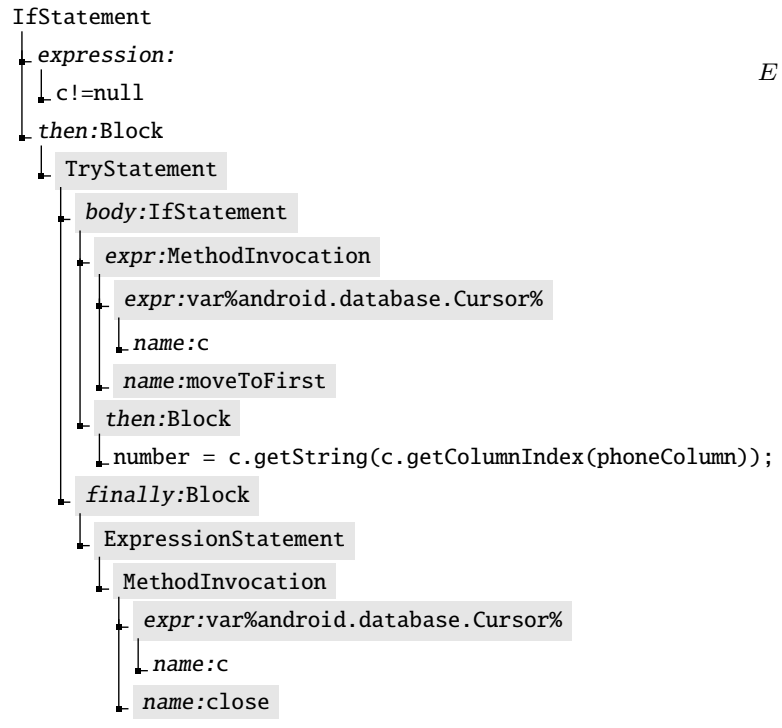

$E \rightarrow$

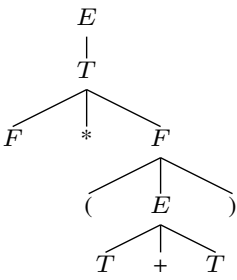

(prob 0.5)

(d)

(c)

Figure 1: Example of code idiom extraction: (a) A snippet from PhoneNumberUtils in android.telephony. (b) A common idiom when handling android. database. Cursor objects, successfully mined by HAGGIs. (c) Eclipse JDT's AST for the code in (a). Shaded nodes are those included in the idiom. (d) An example of a pTSG rule for a simple expression grammar. See text for more details.

types of patterns that are inferred are essentially sequences, or sometimes finite state machines, of method invocations. Although API patterns are valuable, idiom mining is markedly different, because idioms have syntactic structure. For example, current API mining approaches cannot find patterns such as a library with a Tree class that requires special iteration logic, or a Java library that requires the developer to free resources within a finally block. This is exactly the type of pattern that HaGGIs identifies.

\section{MINING CODE IDIOMS}

In this section, we introduce the technical framework that is required for HAGGIS, ${ }^{3}$ our proposed method for the idiom mining problem. At a high level, we approach the problem of mining source code idioms as that of inferring of commonly reoccurring fragments in ASTs. We apply recent advanced techniques from statistical NLP $[10,42]$, but we need to explain them in some detail to justify why they are appropriate for this software engineering task, and why simpler methods would not be effective.

We will build up step by step. First, we will describe our representation of idioms. In particular, we describe a family of probability distributions over ASTs which are called probabilistic tree substitution grammars (pTSGs). A pTSG is essentially a probabilistic context free grammar (PCFG) with the addition of special rules that insert a tree fragment all at once.

Second, we describe how we discover idioms. We do this by learning a pTSG that best explains a large quantity of existing source code. We consider as idioms the tree fragments that appear in the learned pTSG. We learn the pTSG using a powerful general framework called nonparametric Bayesian methods. Nonparametric Bayes provides a principled theoretical framework for automatically inferring how complex a model should be from data. Every time we add a new fragment rule to the PTSG, we are adding a new parameter to the model (the rule's probability of appearing), and the number of potential fragments that we could add is infinite. This creates a

${ }^{3}$ Holistic, Automatic Gathering of Grammatical Idioms from Software. risk that by adding a large number a fragments we could construct a model with too many parameters, which would be likely to overfit the training data. Nonparametric Bayesian methods provide a way to tradeoff the model's fit to the training set with the model's size when the maximum size of the model is unbounded.

It is also worth explaining why we employ probabilistic models here, rather than a standard deterministic CFG. Probabilities provide a natural quantitative measure of the quality of a proposed idiom: A proposed idiom is worthwhile only if, when we include it into a pTSG, it increases the probability that the pTSG assigns to the training corpus. This encourages the method to avoid identifying idioms that are frequent but boring.

At first, it may seem odd that we apply grammar learning methods here, when of course the grammar of the programming language is already known. We clarify that our aim is not to re-learn the known grammar, but rather to learn probability distributions over parse trees from the known grammar. These distributions will represent which rules from the grammar are used more often, and, crucially, which sets of rules tend to be used contiguously.

\subsection{Probabilistic Grammars}

A probabilistic context free grammar (PCFG) is a simple way to define a distribution over the strings of a context-free language. A PCFG is defined as $G=(\Sigma, N, S, R, \Pi)$, where $\Sigma$ is a set of terminal symbols, $N$ a set of nonterminals, $S \in N$ is the root nonterminal symbol and $R$ is a set of productions. Each production in $R$ has the form $X \rightarrow Y$, where $X \in N$ and $Y \in(\Sigma \cup N)^{*}$. The set $\Pi$ is a set of distributions $P(r \mid c)$, where $c \in N$ is a non-terminal, and $r \in R$ is a rule with $c$ on its left-hand side. To sample a tree from a PCFG, we recursively expand the tree, beginning at $S$, and each time we add a non-terminal $c$ to the tree, we expand $c$ using a production $r$ that is sampled from the corresponding distribution $P(r \mid c)$. The probability of generating a particular tree $T$ from this procedure is the product over all rules that are required to generate $T$. The probability $P(x)$ of a string $x \in \Sigma^{*}$ is the sum of the probabilities of the trees $T$ that yield $x$, that is, we simply consider $P(x)$ as a marginal distribution of $P(T)$. 
Tree Substitution Grammars A tree substitution grammar (TSG) is a simple extension to a CFG, in which productions expand into tree fragments rather than simply into a list of symbols. Formally, a TSG is also a tuple $G=(\Sigma, N, S, R)$, where $\Sigma, N, S$ are exactly as in a CFG, but now each production $r \in R$ takes the form $X \rightarrow \mathcal{T}_{X}$, where $\mathcal{T}_{X}$ is a fragment. To produce a string from a TSG, we begin with a tree containing only $S$, and recursively expand the tree in a manner exactly analogous to a CFG - the only difference is that some rules can increase the height of the tree by more than 1 . A probabilistic tree substitution grammar (pTSG) $G[10,42]$ augments a TSG with probabilities, in an analogous way to a PCFG. A pTSG is defined as $G=(\Sigma, N, S, R, \Pi)$ where $\Sigma$ is a set of terminal symbols, $N$ a set of non terminal symbols, $S \in N$ is the root nonterminal symbol, $R$ is a set of tree fragment productions. Finally, $\Pi$ is a set of distributions $P_{T S G}\left(\mathcal{T}_{X} \mid X\right)$, for all $X \in N$, each of which is a distribution over the set of all rules $X \rightarrow \mathcal{T}_{X}$ in $R$ that have left-hand side $X$.

The key reason that we use pTSGs for idiom mining is that each tree fragment $\mathcal{T}_{X}$ can be thought of as describing a set of contextfree rules that are typically used in sequence. This is exactly what we are trying to discover in the idiom mining problem. In other words, our goal will be to induce a pTSG in which every tree fragment represents a code idiom if the fragment has depth greater than 1 , or a rule from the language's original grammar if the depth equals 1 . As a simple example, consider the PCFG

$$
\begin{array}{llll}
E \rightarrow E+E & (\text { prob 0.7) } & T \rightarrow F * F & \text { (prob 0.6) } \\
E \rightarrow T & (\text { prob 0.3) } & T \rightarrow F & \text { (prob 0.4) } \\
F \rightarrow(E) & (\text { prob 0.1) } & F \rightarrow i d & \text { (prob 0.9), }
\end{array}
$$

where $E, T$, and $F$ are non-terminals, and $E$ the start symbol. Now, suppose that we are presented with a corpus of strings from this language that include many instances of expressions like $i d *(i d+i d)$ and $i d *(i d+(i d+i d)$ ) (perhaps generated by a group of students who are practicing the distributive law). Then, we might choose to add a single pTSG rule to this grammar, displayed in Figure 1(d), adjusting the probabilities for that rule and the $E \rightarrow T+T$ and $E \rightarrow T$ rules so that the three probabilities sum to 1 . Essentially, this allows us to a represent a correlation between the rules $E \rightarrow$ $T+T$ and $T \rightarrow F * F$.

Finally, note that every CFG can be written as a TSG where all productions expand to trees of depth 1 . Conversely, every TSG can be converted into an equivalent CFG by adding extra non-terminals (one for each TSG rule $X \rightarrow \mathcal{T}_{X}$ ). So TSGs are, in some sense, fancy notation for CFGs. This notation will prove very useful, however, when we describe the learning problem next.

\subsection{Learning TSGs}

Now we define the learning problem for TSGs that we will consider. First, we say that a pTSG $G_{1}=\left(\Sigma_{1}, N_{1}, S_{1}, R_{1}, P_{1}\right)$ extends a CFG $G_{0}$ if every tree with positive probability under $G_{1}$ is grammatically valid according to $G_{0}$. Given any set $\mathcal{T}$ of tree fragments from $G_{0}$, we can define a pTSG $G_{1}$ that extends $G_{0}$ as follows. First, set $\left(\Sigma_{1}, N_{1}, S_{1}\right)=\left(\Sigma_{0}, N_{0}, S_{0}\right)$. Then, set $R_{1}=R_{C F G} \cup R_{F R A G}$, where $R_{C F G}$ is the set of all rules from $R_{0}$, expressed in the TSG form, i.e., with right-hand sides as trees of depth 1 , and $R_{F R A G}$ is a set of fragment rules $X_{i} \rightarrow \mathcal{T}_{i}$, for all $\mathcal{T}_{i} \in \mathcal{T}$ and where $X_{i}$ is the root of $\mathcal{T}_{i}$.

The grammar learning problem that we consider can be called the $C F G$ extension problem. The input is a set of trees $T_{1} \ldots T_{N}$ from a context-free grammar $G_{0}=\left(\Sigma_{0}, N_{0}, S_{0}, R_{0}\right)$. The CFG extension problem is to learn a pTSG $G_{1}$ that extends $G_{0}$ and is good at explaining the training set $T_{1} \ldots T_{N}$. The notion of "good" is deliberately vague; formalizing it is part of the problem. It should also be clear that we are not trying to learn the CFG for the original programming language - instead, we are trying to identify sequences of CFG rules that commonly co-occur contiguously.

\subsubsection{Why Not Just Count Common Trees?}

A natural first approach to the CFG extension problem is to mine frequent patterns, for example, to return the set of all AST fragments that occur more than a user-specified parameter $M$ times in the training set. This task is called frequent tree mining, and has been the subject of some work in the data mining literature $[25,49$, 54, 55]. Unfortunately, preliminary investigation [31] found that these approaches do not yield good idioms. Instead, the fragments that are returned tend to be small and generic, omitting many details that, to a human eye, are central to the idiom. For example, given the idiom in Figure 1(c), it would be typical for tree mining methods to return a fragment containing the try, if, and finally nodes but not the crucial method call to Cursor. close().

The reason for this is simple: Given a fragment $\mathcal{T}$ that represents a true idiom, it can always be made more frequent by removing one of the leaves, even if that leaf co-occurs often with the rest of the tree. So tree mining algorithms tend to return these shorter trees, resulting in incomplete idioms. This is a general problem with frequent pattern mining: frequent patterns can be boring patterns. To avoid this problem, we need to penalize the method when it chooses not to extend a pattern to include a node that co-occurs frequently. This is what is provided by our probabilistic approach.

A different idea is to use the maximum likelihood principle, that is, to find the pTSG $G_{1}$ that extends $G_{0}$ and maximizes the probability that $G_{1}$ assigns to $T_{1} \ldots T_{N}$. This also does not work. The reason is that a trivial solution is simply to add a fragment rule $E \rightarrow T_{i}$ for every training tree $T_{i}$. This will assign a probability of $1 / N$ to each training tree, which in practice will often be optimal. What is going on here is that the maximum likelihood grammar is overfitting. It is not surprising that this happens: there are an infinite number of potential trees that could be used to extend $G_{0}$, so if a model is given such a large amount of flexibility, overfitting becomes inevitable. What we need is a strong method of controlling overfitting, which the next section provides.

\subsubsection{Nonparametric Bayesian Methods}

At the heart of any application of machine learning is the need to control the complexity of the model. For example, in a clustering task, many standard clustering methods, such as $K$-means, require the user to pre-specify the number of clusters $K$ in advance. If $K$ is too small, then each cluster will be very large and not contain useful information about the data. If $K$ is too large, then each cluster will only contain a few data points, so the again, the cluster centroid will not tell us much about the data set. For the $C F G$ extension problem, the key factor that determines model complexity is the number of fragment rules that we allow for each non-terminal. If we allow the model to assign too many fragments to each non-terminal, then it can simply memorize the training set. But if we allow too few, then the model will be unable to find useful patterns. Nonparametric Bayesian methods provide a powerful and theoretically principled method for managing this trade-off. Although powerful, these methods can be difficult to understand at first. We will not give a detailed tutorial due to space; for a gentle introduction, see Gershman and Blei [16]

To begin, we must first explain Bayesian statistics. Bayesian statistics $[15,37]$ is alternative general framework to classical frequentist statistical methods, such as confidence intervals and hypothesis testing, that allows the analyst to encode prior knowledge about the quantity of interest. The idea behind Bayesian statistics 
is that whenever one wants to estimate an unknown parameter $\theta$ from a data set $x_{1}, x_{2}, \ldots x_{N}$, the analyst should not only treat the data $x_{1} \ldots x_{N}$ as random variables - as in classical statistics - but also $\theta$ as well. To do this, the analyst must choose a prior distribution $P(\theta)$ that encodes any prior knowledge about $\theta$ (if little is known, this distribution can be vague), and then a likelihood $P\left(x_{1} \ldots x_{N} \mid \theta\right)$ that describes a model of how the data is generated given $\theta$. To be clear, the prior and the likelihood are mathematical models of the data, that is, they are mathematical approximations to reality that are designed by the data analyst. Some models are better approximations than others, and more accurate models will yield more accurate inferences about $\theta$.

Once we define a prior and a likelihood, the laws of probability provide only one choice for how to infer $\theta$, namely, via the conditional distribution $P\left(\theta \mid x_{1} \ldots x_{N}\right)$ which is uniquely defined by Bayes' rule. This distribution is called the posterior distribution and encapsulates all of the information that we have about $\theta$ from the data. We can compute summaries of the posterior to make inferences about $\theta$, for example, if we want to estimate $\theta$ by a single vector, we might compute the mean of $P\left(\theta \mid x_{1} \ldots x_{N}\right)$. Although mathematically the posterior distribution is a simple function of the prior and likelihood, in practice it can be very difficult to compute, and approximations are often necessary. To summarize, applications of Bayesian statistics have three steps: first, choose a prior $p(\theta)$; second, choose a likelihood $p\left(x_{1} \ldots x_{N} \mid \theta\right)$, finally, compute $p\left(\theta \mid x_{1} \ldots x_{N}\right)$ using Bayes's rule.

As a simple example, suppose the data $x_{1} \ldots x_{N}$ are real numbers, which we believe to be distributed independently according a Gaussian distribution with variance 1 but unknown mean $\theta$. Then we might choose a prior $p(\theta)$ to be Gaussian with mean 0 and a large variance, to represent the fact that we do not know much about $\theta$ before we see the data. Our beliefs about the data indicate that $p\left(x_{i} \mid \theta\right)$ is Gaussian with mean $\theta$ and variance 1 . By applying Bayes's rule, it is easy to show that $P\left(\theta \mid x_{1} \ldots x_{N}\right)$ is also Gaussian, whose mean is approximately ${ }^{4}$ equal to $N^{-1} \sum_{i} x_{i}$ and whose variance is approximately $1 / N$. This distribution represents a Bayesian's belief about the unknown mean $\theta$, after seeing the data.

Nonparametric Bayesian methods handle the more complex case where the number of parameters is unknown as well. For example, consider a clustering model where, conditioned on the cluster identity, the data is Gaussian, but the number of clusters is unknown. In this case, $\theta$ would be a vector containing the centroid for each cluster, but then, because before we see the data the number of clusters could be arbitrarily large, $\theta$ has unbounded dimension. Nonparametric Bayesian methods focus on developing prior distributions over such infinite dimensional objects, which are then used within Bayesian statistical inference. Bayesian nonparametrics have been the subject of intense research in statistics and in machine learning, with popular models including the Dirichlet process [19] and the Gaussian process [53].

Applying this discussion to the CFG extension problem, what we are trying to infer is a pTSG $T$. So, to apply Bayesian inference, our prior distribution must be a probability distribution over probabilistic grammars. In order to define this distribution, we will need to take a brief digression and define first a distribution $P_{0}(T)$ over fragments from a CFG. Let $G_{0}$ be the known CFG for the programming language in question. We will assume that we have available a PCFG for $G_{0}$, because this can be easily estimated by maximum likelihood from a training corpus; call this distribution $P_{\mathrm{ML}}$. Now, $P_{\mathrm{ML}}$ gives us a distribution over full trees. To get a distribution over

\footnotetext{
${ }^{4}$ The exact value depends on precisely what variance we choose in $p(\theta)$, but the formula is simple.
}

fragments, we include a distribution over tree sizes, yielding

$$
P_{0}(T)=P_{\text {geom }}\left(|T|, p_{\$}\right) \prod_{r \in T} P_{\mathrm{ML}}(r),
$$

where $|T|$ is the size of the fragment $T, P_{\text {geom }}$ is a geometric distribution with parameter $p_{\$}$, and $r$ ranges over the multiset of productions that are used within $T$.

Now we can define a prior distribution over pTSGs. Recall that we can define a pTSG $G_{1}$ that extends $G_{0}$ by specifying a set of tree fragments $\mathcal{F}_{X}$ for each non-terminal $X$. So, to define a distribution over pTSGs, we will define a distribution $P\left(\mathcal{F}_{X}\right)$ over the set of tree fragments rooted at $X$. We need $P\left(\mathcal{F}_{X}\right)$ to have several important properties. First, we need $P\left(\mathcal{F}_{X}\right)$ to have infinite support, that is, it must assign positive probability to all possible fragments. This is because if we do not assign a fragment positive probability in the prior distribution, we will never be able to infer it as an idiom, no matter how often it appears. Second, we want $P\left(\mathcal{F}_{X}\right)$ to exhibit a "rich-get-richer" effect, namely, once we have observed that a fragment $\mathcal{T}_{X}$ occurs many times, we want to be able to predict that it will occur more often in the future.

A natural distribution with these properties is the Dirichlet process (DP). The Dirichlet process has two parameters: a base measure, ${ }^{5}$ in our case, the fragment distribution $P_{0}$, and a concentration parameter $\alpha \in \mathbb{R}^{+}$, which controls the strength of the rich-get-richer effect. Following the stick-breaking representation [46], a Dirichlet process defines a prior distribution over $\mathcal{F}_{X}$ as

$$
\begin{aligned}
\operatorname{Pr}\left[\mathcal{T} \in \mathcal{F}_{X}\right] & =\sum_{k=1_{k-1}}^{\infty} \pi_{k} \delta_{\left\{\mathcal{T}=\mathcal{T}_{k}\right\}} & \mathcal{T}_{k} & \sim P_{0} \\
\pi_{k} & =u_{k} \prod_{j=1}^{\left.1-u_{j}\right)} & & u_{k} \sim \operatorname{Beta}(1, \alpha) .
\end{aligned}
$$

To interpret this, recall that the symbol $\sim$ is read "is distributed as," the Beta distribution is a standard distribution over the set $[0,1]$, and $\delta_{\left\{\mathcal{T}=\mathcal{T}_{k}\right\}}$ is a delta function, i.e., a probability distribution over $\mathcal{T}$ that generates $\mathcal{T}_{k}$ with probability 1 . Intuitively, what is going on here is that a sample from the DP is a distribution over a countably infinite number of fragments $\mathcal{T}_{1}, \mathcal{T}_{2}, \ldots$. Each one of these fragments is sampled independently from the fragment distribution $P_{0}$. To assign a probability to each fragment, we recursively split the interval $[0,1]$ into a countable number of sticks $\pi_{1}, \pi_{2}, \ldots$. The value $\left(1-u_{k}\right)$ defines what proportion of the remaining stick is assigned to the current sample $\mathcal{T}_{k}$, and the remainder is assigned to the infinite number of remaining trees $\mathcal{T}_{k+1}, \mathcal{T}_{k+2}, \ldots$ This process defines a distribution over fragments $\mathcal{F}_{X}$ for each non-terminal $X$, and hence a distribution $P\left(G_{1}\right)$ over the set of all pTSGs that extend $G_{0}$. We will refer to this distribution as a Dirichlet process probabilistic tree substitution grammar (DPpTSG) $[42,10]$.

This process may seem odd for two reasons: (a) each sample from $P\left(G_{1}\right)$ is infinitely large, so we cannot store it exactly on a computer, (b) the fragments from $G_{1}$ are sampled randomly from a PCFG, so there is no reason to think that they should match real idioms. Fortunately, the answer to both these concerns is simple. We are not interested in the fragments that exist in the prior distribution, but rather of those in the posterior distribution. More formally, the DP provides us with a prior distribution $G_{1}$ over pTSGs. But $G_{1}$ itself, like any pTSG, defines a distribution $P\left(T_{1}, T_{2}, \ldots T_{N} \mid G_{1}\right)$ over the training set. So, just as in the parametric case, we can apply Bayes's rule to obtain a posterior distribution $P\left(G_{1} \mid T_{1}, T_{2}, \ldots T_{N}\right)$. It can be shown that this distribution is also a DPpTSG, and, amazingly,

${ }^{5}$ The base measure will be a probability measure, so for our purposes, we can think of this as a fancy word for "base distribution". 


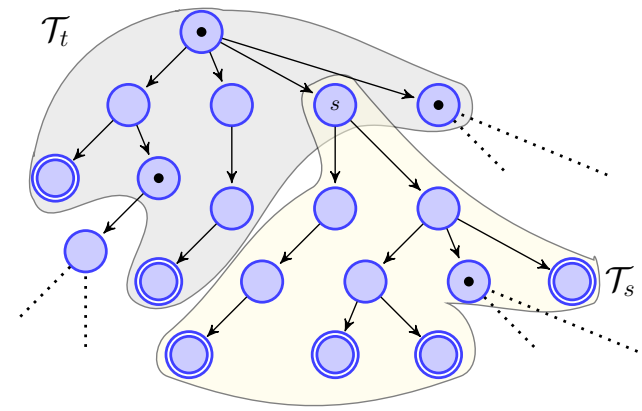

Figure 2: Sampling an AST. Dots show the points where the tree is split (i.e., $z_{t}=1$ ). Terminal nodes have double border.

that this posterior DPpTSG can be characterized by a finite set of fragments $\mathcal{F}_{X}^{\prime}$ for each non-terminal. It is these fragments that we will identify as code idioms (Section 4).

\subsubsection{Inference}

Now that we have defined a posterior distribution over probabilistic grammars, we now need to describe how to compute this distribution. Unfortunately, the posterior distribution cannot be computed exactly, so we resort to approximations. The most commonly used approximations in the literature are based on Markov chain Monte Carlo (MCMC), which we explain below. But first, we make one more observation about pTSGs. All of the pTSGs that we consider are extensions of an unambiguous base CFG $G_{0}$. This means that given a source file $F$, we can separate the pTSG parsing task into two steps: first, parse $F$ using $G_{0}$, resulting in a CFG tree $T$; second, group the nodes in $T$ according to which fragment rule in the pTSG was used to generated them. We can represent this second task as a tree of binary variables $z_{s}$ for each node $s$. These variables indicate whether $s$ is the root of a new fragment $\left(z_{s}=1\right)$, or if $s$ is part of the same fragment as its parent $\left(z_{s}=0\right)$. Essentially, the variables $z_{s}$ show the boundaries of the inferred tree patterns; see Figure 2 for an example. Conversely, even if we don't know what fragments are in the grammar, given a training corpus that has been parsed in this way, we can use the $z_{s}$ variables to read off what fragments must have been in the pTSG.

With this representation in hand, we are now ready to present an MCMC method for sampling from the posterior distribution over grammars, using a particular method called Gibbs sampling. Gibbs sampling is an iterative method, which starts with an initial value for all of the $z$ variables, and then updates them one at a time. At each iteration, the sampler visits every tree node $t$ of every tree in the training corpus, and samples a new value for $z_{t}$. Let $s$ be the parent of $t$. If we choose $z_{t}=1$, we can examine the current values of the $z$ variables to determine the tree fragment $\mathcal{T}_{t}$ that contains $t$ and the fragment $\mathcal{T}_{s}$ for $s$, which must be disjoint. On the other hand, if we set $z_{t}=0$, then $s$ and $t$ will belong to the same fragment, which will be exactly $\mathcal{T}_{\text {join }}=\mathcal{T}_{s} \cup \mathcal{T}_{t}$. Now, we set $z_{t}$ to 0 with probability

$$
P\left(z_{t}=0\right)=\frac{P_{\text {post }}\left(\mathcal{T}_{\text {join }}\right)}{P_{\text {post }}\left(\mathcal{T}_{\text {join }}\right)+P_{\text {post }}\left(\mathcal{T}_{s}\right) P_{\text {post }}\left(\mathcal{T}_{t}\right)} .
$$

where

$$
P_{\text {post }}(\mathcal{T})=\frac{\operatorname{count}(\mathcal{T})+\alpha P_{0}(\mathcal{T})}{\operatorname{count}(h(\mathcal{T}))+\alpha}
$$

$h$ returns the root of the fragment, and count returns the number of times that a tree occurs as a fragment in the corpus, as determined by the current values of $z$. Intuitively, what is happening here is that if the fragments $\mathcal{T}_{s}$ and $\mathcal{T}_{t}$ occur very often together in the corpus, relative to the number of times that they occur independently, then we are more likely to join them into a single fragment.

It can be shown that if we repeat this process for a large number of iterations, eventually the resulting distribution over fragments will converge to the posterior distribution over fragments defined by the DPpTSG. It is these fragments that we return as idioms.

We present the Gibbs sampler because it is a useful illustration of MCMC, but in practice we find that it converges too slowly to scale to large code bases. Instead we use the type-based MCMC sampler of Liang et al. [33] (details omitted).

\section{SAMPLING A TSG FOR CODE}

In this section, we describe a set of necessary transformations to ASTs and pTSGs to adapt these general methods specifically to the task of inferring code idioms.

AST Transformation For each . java file we use the Eclipse JDT [12] to extract its AST - a tree structure of ASTNode objects. Each ASTNode object contains two sets of properties: simple properties - such as the type of the operator, if ASTNode is an infix expression - and structural properties that contain zero or more child ASTNode objects. First, we construct the grammar symbols by mapping each ASTNode's type and simple properties into a single (terminal or non-terminal) symbol. The transformed tree is then constructed by mapping the original AST into a tree whose nodes are annotated with the symbols. Each node's children are grouped by property. The transformed trees may contain nodes that have more than two children for a single property (e.g. Block). This induces unnecessary sparsity in the CFG and TSG rules. To reduce this, we perform tree binarization. This process - common in NLP — transforms the original tree into a binary tree by adding dummy nodes, making the data less sparse. It also helps us capture idioms in sequential statements. Note that binarization is performed per structural property only when it contains more than two children, while a node will generally have more than two children across all its structural properties.

One final hurdle for learning meaningful code idioms are variable names. Since variable names are mostly project or class specific we abstract them introducing an intermediate MetaVariable node between the SimpleName node containing the string representation of the variable name and its parent node. MetaVariable nodes are also annotated with the type of the variable they are abstracting. This provides the pTSG with the flexibility to either exclude or include variable names as appropriate. For example, in the snippet of Figure 1(a) by using metavariables, we are able to learn the idiom in Figure 1(b) without specifying the name of the Cursor object by excluding the SimpleName nodes from the fragment. Alternatively, if a specific variable name is common and idiomatic, such as the $i$ in a for loop, the pTSG can choose to include SimpleName in the extracted idiom, by merging it with its parent MetaVariable node.

Training TSGs and Extracting Code Idioms Training a pTSG happens offline, during a separate training phase. After training the pTSG, we then extract the mined code idioms which then can be used for any later visualization. In other words, a user of a HAGGIS IDE tool would never need to wait for an MCMC method to finish. The output of an MCMC method is a series of (approximate) samples from the posterior distribution, each of which in our case, is a single pTSG. These sampled pTSGs need to be post-processed to extract a single, meaningful set of code idioms. First, we aggregate the MCMC samples after removing the first few samples as burnin, which is standard methodology for applying MCMC. Then, to extract idioms from the remaining samples, we merge all samples' tree fragments into a single multiset. We prune this multiset by 


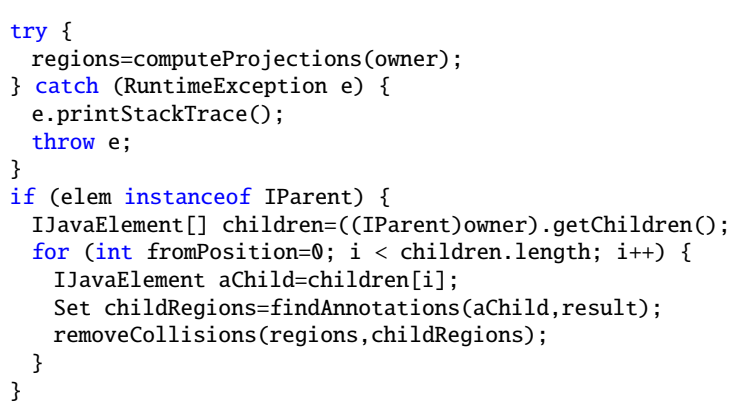

Figure 3: Synthetic code randomly generated from a posterior pTSG. The pTSG produces syntactically correct and locally consistent code. This effect allows us to infer code idioms. As expected, the pTSG cannot capture higher level information, such as variable binding.

removing all tree fragments that have been seen less than $c_{\text {min }}$ times. We also prune fragments that have fewer that $n_{\min }$ nodes to remove trivial idioms. Finally, we convert the remaining fragments back to Java code. The leaf nodes of the fragments that contain non-terminal symbols represent metavariables and are converted to the appropriate symbol that is denoted by a $\$$ prefix.

Additionally, to assist the sampler in inducing meaningful idioms, we prune any import statements from the corpus, so that they cannot be mined as idioms. We also exclude some nodes from sampling, fixing $z_{i}=0$ and thus forcing some nodes to be un-splittable. Such nodes include method invocation arguments, qualified and parametrized type node children, non-block children of while, for and if statement nodes, parenthesized, postfix and infix expressions and variable declaration statements.

\section{CODE SNIPPET EVALUATION}

We take advantage of the omnipresence of idioms in source code to evaluate HAGGIS on popular open source projects. We restrict ourselves to the Java programming language, due to the high availability of tools and source code. We emphasize, however, that HaGGIs is language agnostic. Before we get started, an interesting way to get an intuitive feel for any probabilistic model is simply to draw samples from it. Figure 3 shows a code snippet that we synthetically generated by sampling from the posterior distribution over code defined by the pTSG. One can observe that the pTSG is learning to produce idiomatic and syntactically correct code, although — as expected - the code is semantically inconsistent.

Methodology We use two evaluation data sets comprised of Java open-source code available on GitHub. The Projects data set (Figure 4) contains the top 13 Java GitHub projects whose repository is at least $100 \mathrm{MB}$ in size according to the GitHub Archive [17]. To determine popularity, we computed the $z$-score of forks and watchers for each project. The normalized scores were then averaged to retrieve each project's popularity ranking. The second evaluation data set, LIBRARY (Figure 5), consists of Java classes that import (i.e. use) 15 popular Java libraries. For each selected library, we retrieved from the Java GitHub Corpus [2] all files that import that library but do not implement it. We split both data sets into a train and a test set, splitting each project in Projects and each library file set in Library into a train $(70 \%)$ and a test $(30 \%)$ set. The Projects will be used to mine project-specific idioms, while the LIBRARY will be used to mine idioms that occur across libraries.

To extract idioms we run MCMC for 100 iterations for each of the projects in PROJECTs and each of the library file sets in LIBRARY, using the first 75 iterations as burn-in. For the last 25 iterations, we aggregate a sample posterior pTSG and extract idioms as detailed

\begin{tabular}{lrrrrl}
\hline Name & Forks & Stars & Files & Commit & Description \\
\hline arduino & 2633 & 1533 & 180 & 2757691 & Electronics Prototyping \\
atmosphere & 1606 & 370 & 328 & a0262bf & WebSocket Framework \\
bigbluebutton & 1018 & 1761 & 760 & e3b6172 & Web Conferencing \\
elasticsearch & 5972 & 1534 & 3525 & ad547eb & REST Search Engine \\
grails-core & 936 & 492 & 831 & $15 f 9114$ & Web App Framework \\
hadoop & 756 & 742 & 4985 & f68ca74 & Map-Reduce Framework \\
hibernate & 870 & 643 & 6273 & d28447e & ORM Framework \\
libgdx & 2903 & 2342 & 1985 & Oc6a387 & Game Dev Framework \\
netty & 2639 & 1090 & 1031 & $3 f 53 \mathrm{ba} 2$ & Net App Framework \\
storm & 1534 & 7928 & 448 & cdb116e & Distributed Computation \\
vert.x & 2739 & 527 & 383 & $9 \mathrm{ff} 79416$ & Application platform \\
voldemort & 347 & 1230 & 936 & 9ea2e95 & NoSQL Database \\
wildfly & 1060 & 1040 & 8157 & Q43d7d5 & Application Server \\
\hline
\end{tabular}

Figure 4: Projects data set used for in-project idiom evaluation. Projects in alphabetical order.

\begin{tabular}{lrl}
\hline Package Name & Files & Description \\
\hline android.location & 1262 & Android location API \\
android.net.wifi & 373 & Android WiFi API \\
com.rabbitmq & 242 & Messaging system \\
com.spatial4j & 65 & Geospatial library \\
io.netty & 65 & Network app framework \\
opennlp & 202 & NLP tools \\
org.apache.hadoop & 8467 & Map-Reduce framework \\
org.apache.lucene & 4595 & Search Server \\
org.elasticsearch & 338 & REST Search Engine \\
org.eclipse.jgit & 1350 & Git implementation \\
org.hibernate & 7822 & Persistence framework \\
org.jsoup & 335 & HTML parser \\
org.mozilla.javascript & 1002 & JavaScript implementation \\
org.neo4j & 1294 & Graph database \\
twitter4j & 454 & Twitter API \\
\hline
\end{tabular}

Figure 5: LIBRARY data set for cross-project idiom evaluation. Each API file set contains all class files that import a class belonging to the respective package or one of its subpackages.

in Section 4. A threat to the validity of the evaluation using the aforementioned data sets is the possibility that the data sets are not representative of Java development practices, containing solely opensource projects from GitHub. However, the selected data sets span a wide variety of domains, including databases, messaging systems and code parsers, diminishing any such possibility. Furthermore, we perform an extrinsic evaluation on source code found on a popular online Q\&A website, Stack Overflow.

Evaluation Metrics We compute two metrics on the test corpora. These metrics resemble precision and recall in information retrieval but are adjusted to the code idiom domain. We define idiom coverage as the percent of source code AST nodes that matches any of the mined idioms. Coverage is thus a number between 0 and 1 indicating the extent to which the mined idioms exist in a piece of code. We define idiom set precision as the percentage of the mined idioms that also appear in the test corpus. Using these two metrics, we tune the concentration parameter of the DPpTSG model by using android.net.wifi as a validation set, yielding $\alpha=1$.

\subsection{Top Idioms}

Figure 6 shows the top idioms mined in the LIBRARY data set, ranked by the number of files in the test sets where each idiom has appeared in. The reader will observe their immediate usefulness. Some idioms capture how to retrieve or instantiate an object. For example, in Figure 6, the idiom 6a captures the instantiation of a message channel in RabbitMQ, 6q retrieves a handle for the Hadoop file system, 6e builds a SearchSourceBuilder in Elasticsearch and 61 retrieves a URL using JSoup. Other idioms capture important transactional properties of code: idiom $6 \mathrm{~h}$ demonstrates proper use 
channel=connection. createChannel();

(a)

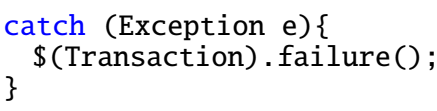

(d)

Location.distanceBetween(
\$(Location).getLatitude(),
\$(Location).getLongitude(),
\$...);

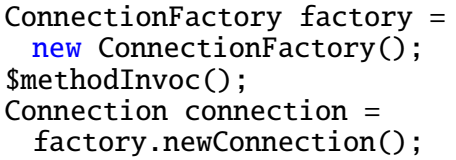

(j)

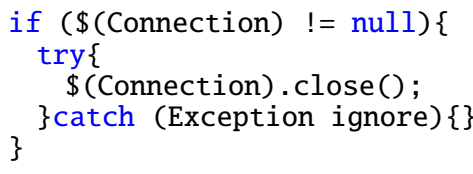

(m)

$\operatorname{try}\{$

Session session

=HibernateUtil \$BODY\$

currentSession()

\}catch (HibernateException e) \{ \} throw new DaoException(e);

(p)
Elements \$name $=\$$ (Element). select (\$StringLit);

(b)

SearchSourceBuilder builder= getQueryTranslator() . build( $\$($ ContentIndexQuery));

(e)

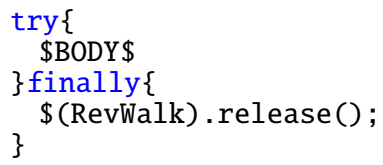

(h) (i)

Transaction $\mathrm{tx}=$ ConnectionFactory . getDatabase(). beginTx();

(c)

LocationManager \$name = (LocationManager)getSystemService( Context.LOCATION_SERVICE);

(f)

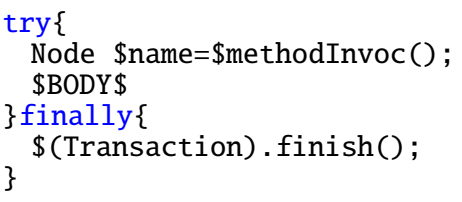

Document doc=J soup. connect (URL). userAgent ("Mozilla") header ("Accept", "text/html"). $\operatorname{get}()$;

(1)

Traverser traverser $=\$$ (Node) . traverse (); for (Node \$name: traverser) \{ \$BODY\$ \}

(n)

\author{
FileSystem \$name \\ $=$ FileSystem.get ( \\ $\$($ Path) . toUri(), conf);
}

\author{
(token=\$(XContentParser) \\ . nextToken()) \\ ! = XContentParser \\ .Token.END_OBJECT
}

(q)

(r)

Figure 6: Top cross-project idioms for LibraRy projects (Figure 4). Here we include idioms that appear in the test set files. We rank them by the number of distinct files they appear in and restrict into presenting idioms that contain at least one library-specific (i.e. API-specific) identifier. The special notation \$ (TypeName) denotes the presence of a variable whose name is undefined. \$BODY\$ denotes a user-defined code block of one or more statements, \$name a freely defined (variable) name, \$methodInvoc a single method invocation statement and \$ifstatement a single if statement. All the idioms have been automatically identified by HAGGIS

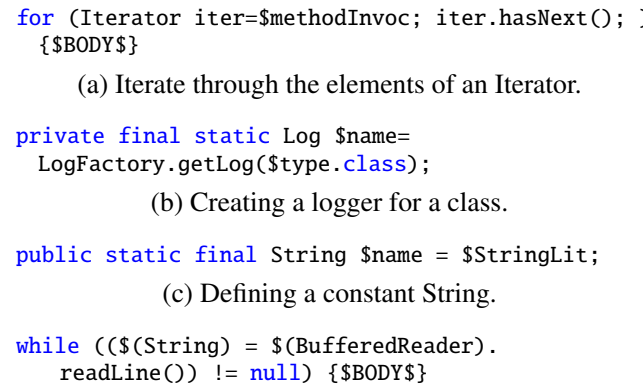

(a) Iterate through the elements of an Iterator.

private final static Log \$name=

LogFactory.getLog (\$type.class);

(b) Creating a logger for a class.

public static final String \$name $=\$$ StringLit;

(c) Defining a constant String.

while $((\$($ String $)=\$($ BufferedReader $)$. readLine ()) $!=$ null) $\{\$$ BODY $\$$

(d) Looping through lines from a BufferedReader.

Figure 7: Sample language-specific idioms. \$StringLit denotes a user-defined string literal, \$name a (variable) name, \$methodInvoc a method invocation statement, \$ifstatement an if statement and \$BODY\$ a code block.

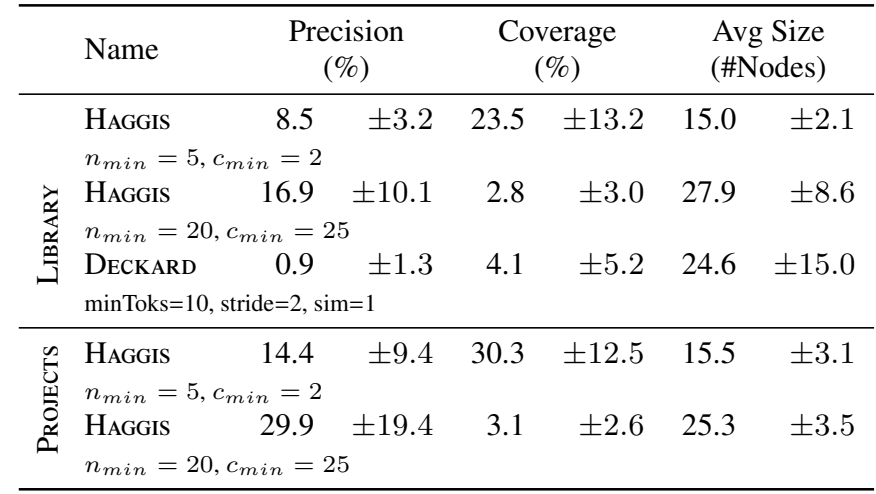

Figure 8: Average and standard deviation of performance in LIBRARY test set. Standard deviation across projects. 


\begin{tabular}{lrr}
\hline Test Corpus & Coverage & Precision \\
\hline Stack Overflow & $31 \%$ & $67 \%$ \\
PROJECTs & $22 \%$ & $50 \%$ \\
\hline
\end{tabular}

Figure 9: Extrinsic evaluation of mined idioms from Library.

of the memory-hungry RevWalk object in JGit and $6 \mathrm{i}$ is a transaction idiom in Neo4J. Other idioms capture common error handling, such as $6 \mathrm{~d}$ for Neo4J and $6 \mathrm{p}$ for a Hibernate transaction. Finally, some idioms capture common operations, such as closing a connection in Netty (6m), traversing through the database nodes (6n), visiting all AST nodes in a JavaScript file in Rhino (6k) and computing the distance between two locations (6g) in Android. The reader may observe that these idioms provide a meaningful set of coding patterns for each library, capturing semantically consistent actions that a developer is likely to need when using these libraries.

In Figure 7 we present a small set of general Java idioms mined across all data sets by HaGGIs. These idioms represent frequently used patterns that could be included by default in tools such as Eclipse's SnipMatch [43] and IntelliJ's live templates [23]. These include idioms for defining constants (Figure 7c), creating loggers (Figure 7b) and iterating through an iterable (Figure 7a).

We now quantitatively evaluate the mined idiom sets. Figure 8 shows idiom coverage, idiom set precision and the average size of the matched idioms in the test sets of each data set. We observe that HAGGIS achieves better precision and coverage in Projects than LiBRARY. This is expected since code idioms recur more often within a project than across disparate projects. This effect may be partially attributed to the small number of people working in a project and partially to project-specific idioms. Figure 8 also gives an indication of the trade-offs we can achieve for different $c_{\min }$ and $n_{\min }$.

\subsection{Code Cloning vs Code Idioms}

Previously, we argued that code idioms differ significantly from code clones. We now show this by using a cutting-edge clone detection tool: Deckard [24] is a state-of-the-art tree-based clonedetection tool that uses an intermediate vector representation to detect similarities. To extract code idioms from the code clone clusters that DECKARD computes, we retrieve the maximal common subtree of each cluster, ignoring patterns that are less than $50 \%$ of the original size of the tree.

We run DecKard on the validation set with multiple parameters (stride $\in\{0,2\}$, similarity $\in\{0.95,1.0\}$, minToks $\in\{10,20\}$ ) and picked those that achieve the best combination of precision and coverage. These parameters would be plausible choices if one would try to mine idioms with a clone detection tool. Figure 8 shows precision, coverage and average idiom size (in number of nodes) of the patterns found through Deckard and HagGis. Haggis found larger and higher coverage idioms, since clones seldom recur across projects. The differences in precision and coverage are statistically significant (paired $t$-test; $p<0.001$ ). We note that the overlap in the patterns extracted by DeCKARD and HaGGIS is small $(<0.5 \%)$.

These results are not a criticism of DECKARD - which is a highquality, state-of-the-art code clone detection tool — but rather show that the task of code clone detection is different from code idiom mining. Code clone detection - even when searching for gapped clones - is concerned with finding pieces of code that are not necessarily frequent but are maximally identical. In contrast, idiom mining is concerned with finding very common tree fragments that trade off between pattern size and frequency.

\subsection{Extrinsic Evaluation of Mined Idioms}

Now, we evaluate HAGGIS extrinsically on a data set of Stack
Packages

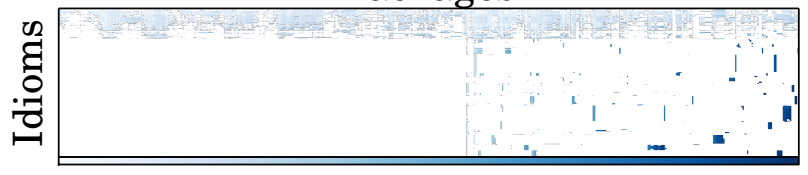

Figure 10: Lift (i.e. co-occurrence) between packages and code idioms. Rows show the correlation between packages and idioms. Darker blue color shows higher correlation. Generic/language idioms are found on the top and package-specific at the dark blocks on the right. Idioms and packages shown only for android. location, android.net.wifi and org.hibernate for brevity.

Overflow questions [4]. Stack Overflow is a popular Q\&A site for programming-related questions. The questions and answers often contain code snippets, which are representative of general development practice and are usually short, concise and idiomatic, containing only essential pieces of code. Our hypothesis is that snippets from Stack Overflow are more idiomatic than typical code, so if HAGGIs idioms are meaningful, they will occur more commonly in code snippets from Stack Overflow than in typical code.

To test this, we first extract all code fragments in questions and answers tagged as java or android, filtering only those that can be parsed by Eclipse JDT [12]. We further remove snippets that contain less than 5 tokens. After this process, we have 108,407 partial Java snippets. Then, we create a single set of idioms, merging all those found in LIBRARY and removing any idioms that have been seen in less than five files in the LiBRARY test set. We end up with small but high precision set of idioms across all APIs in LiBrARY.

Figure 9 shows precision and coverage of HAGGIs's idioms comparing Stack Overflow, Library and Projects. Using the Library idioms, we achieve a coverage of $31 \%$ and a precision of $67 \%$ on Stack Overflow, compared to a much smaller precision and coverage in Projects. This shows that the mined idioms are more frequent in Stack Overflow than in a "random" set of projects. Since we expect that Stack Overflow snippets are more highly idiomatic than average projects' source code, this provides strong indication that HAGGIs has mined a set of meaningful idioms. We note that precision depends highly on the popularity of LiBRARY's libraries. For example, because Android is one of the most popular topics in Stack Overflow, when we limit the mined idioms to those found in the two Android libraries, HAGGIS achieves a precision of $96.6 \%$ at a coverage of $21 \%$ in Stack Overflow. This indicates that HagGis idioms are widely used in development practice.

Eclipse Snipmatch To further evaluate HAGGIs, we submitted a set of idioms to Eclipse Snipmatch [43]. Snipmatch currently contains about 100 human-created code snippets. Currently only JRE, SWT and Eclipse specific snippets are being accepted. Upon discussion with the community, we mined a set of idioms specifically for SWT, JRE and Eclipse. Some of the HAGGIs mined idioms already existed in Snipmatch. Of the remaining idioms, we manually translated 27 idioms into JFace templates, added a description and submitted them for consideration. Five of these were merged as is, four were rejected because of unsupported features/libraries in Snipmatch (but might be added in the future), one was discarded as a bad practice that nevertheless appeared often in our data, and one more was discarded because it already existed in Snipmatch. Finally, another snippet was rejected to allow Snipmatch "to keep the snippets balanced, i.e., cover more APIs equally well". The remaining fifteen were still under consideration at the time of writing. This provides informal evidence that HaGGIs mines useful idioms that other developers find useful. Nevertheless, this experience also highlights that, as with any data-driven method, the idioms mined will also reflect any old 


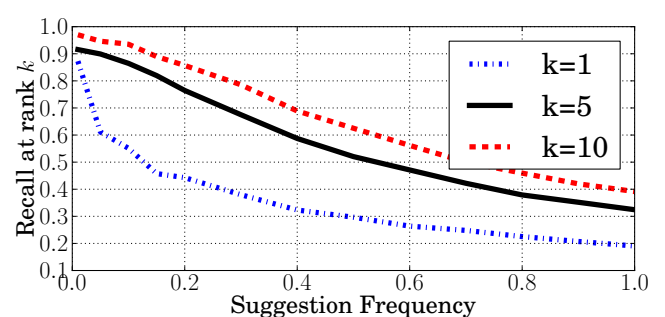

Figure 11: Recall at rank $k$ for code idiom suggestion.

or deprecated coding practices in the data.

\subsection{Idioms and Code Libraries}

As a final evaluation of the mined code idioms' semantic consistency, we now show that code idioms are highly correlated with the imported packages of a Java file. We merge the idioms across our LIBRARY projects and visualize the lift among code idioms and import statements. Lift, commonly used in association rule mining, measures how dependent the co-appearance of two elements is. For each imported package $p$, we compute lift $l$ of the code idiom $t$ as $l(p, t)=P(p, t) /(P(p) P(t))$ where $P(p)$ is the probability of importing package $p, P(t)$ is the probability of the appearance of code idiom $t$ and $P(p, t)$ is the probability that package $p$ and idiom $t$ appear together. $l(p, t)$ is higher as package $p$ and idiom $t$ are more correlated, i.e., their appearance is not independent.

Figure 10 shows a matrix of the lift of the top idioms and packages. We show the top 300 most frequent packages in the training set and their highest correlating code idioms, along with the top 100 most frequent idioms in LiBRARY. Each row represents a single code idiom and each column a single package. At the top, one can see idioms that do not depend strongly on the package imports. These are generic idioms (e.g., Figure 7c) that do not correlate significantly with any package. We can also observe dark blocks of packages and idioms. Those represent library or project-specific idioms that co-appear frequently. This provides additional evidence that $\mathrm{HAG}_{\mathrm{AG}}$ GIS finds meaningful idioms since, as expected, some idioms are common throughout Java, while others are API or project-specific.

Suggesting idioms To further demonstrate the semantic consistency of the HAGGIS idioms, we present a preliminary approach to suggesting idioms based on package imports. We caution that our goal here is to develop an initial proof of concept, not the best possible suggestion method. First, we score each idiom $\mathcal{T}_{i}$ by computing $s\left(\mathcal{T}_{i} \mid \mathbb{I}\right)=\max _{p \in \mathbb{I}} l\left(p, \mathcal{T}_{i}\right)$ where $\mathbb{I}$ is the set of all imported packages. We then return a ranked list $\mathbb{T}_{\mathbb{I}}=\left\{\mathcal{T}_{1}, \mathcal{T}_{2}, \ldots\right\}$ such that for all $i<j, s\left(\mathcal{T}_{i}, \mathbb{I}\right)>s\left(\mathcal{T}_{j}, \mathbb{I}\right)$. Additionally, we use a threshold $s_{t h}$ to control the precision of the returned suggestions, showing only those idioms $t_{i}$ that have $s\left(\mathcal{T}_{i}, \mathbb{I}\right)>s_{t h}$. Thus, we are only suggesting idioms where the level of confidence is higher than $s_{t h}$. This parameter controls suggestion frequency, i.e. the percent of the times where we present at least one code idiom.

To evaluate HAGGIS's idiom suggestions, we use the LIBRARY idioms mined from the train set and compute the recall-at-rank- $k$ on the LIBRARY's test set. Recall-at-rank- $k$ evaluates HaGGIs's ability to return at least one code idiom for each test file. Figure 11 shows that for suggestion frequency of $20 \%$ we achieve a recall of $76 \%$ at rank $k=5$, meaning that in the top 5 results we return at least one relevant idiom $76 \%$ of the time. This result shows the quality of the mined idioms, suggesting that HaGGIs can provide a set of meaningful suggestions to developers by solely using the code's imports. Further improvements in performance can be achieved by using advanced classification methods, which we leave to future work, and will enable an IDE side-pane with suggested code idioms.

\section{RELATED WORK}

Source code has been shown to be highly repetitive [14], suggesting that statistical NLP methods could be promising for code analysis. $N$-gram language models have been used to improve code autocompletion performance [2, 18, 39], learn coding conventions [3] and find syntax errors [8]. Models of the tree structure of the code have also been studied with the aim of generating programs by example [35] and modeling source code [34]. However, none of this work has tried to extract non-sequential patterns in code or mine tree fragments. The only work that we are aware of that uses language models for detecting textual patterns in code is Jacob and Tairas [21], who use $n$-grams to autocomplete code templates.

Code clones [6, 11, 26, 27, 28, 32, 44, 45] are related to idiom mining, since they aim to find blocks of highly similar code. Code clone detection using ASTs has also been studied extensively [7, 13, 24, 30]. For a survey of clone detection methods, see Roy et al. $[44,45]$. In contrast to clone detection, as we noted in Section 5, code idiom mining searches for frequent, rather than maximally identical subtrees. It is worth noting that code clones have been found to have a positive effect on maintenance [27, 28]. Another related area is API mining [1, 20, 56, 51]. However, this problem is also significantly different from code idiom mining because it tries to mine sequences or graphs [38] of API method calls, usually ignoring most features of the language. This difference should be evident from the sample code idioms in Figure 6.

Within the data mining literature, there has been a series of work on frequent tree mining algorithms [25, 49, 54, 55], which focuses on finding subtrees that occur often in a database of trees. However, as described in Section 3.2.1, these have the difficulty that frequent trees are not always interesting trees, a difficulty which our probabilistic approach addresses in a principled way. Finally, as described previously, Bayesian nonparametric methods are a widely researched area in statistics and machine learning [19, 16, 48, 41], which have also found many applications in NLP [47, 10, 42].

\section{DISCUSSION \& CONCLUSIONS}

We presented HAGGIs, a system for automatically mining highquality code idioms. The idioms discovered include project, API, and language specific idioms. One interesting direction for future work is the question of why code idioms arise and their effect on the software engineering process. It may be that there are "good" and "bad" idioms. "Good" idioms could arise as an additional abstraction over programming languages helping developers communicate more clearly their intention. "Bad" idioms may compensate for deficiencies of a programming language or an API. For example, the "multi-catch" statement in Java 7 [40] was designed to remove the need for an idiom that consisted of a sequence of catch statements with identical bodies. However, it may be argued that other idioms, such as the ubiquitous for (int $i=0 ; i<n ; i++$ ) aid code understanding. A formal study about the differences between these types of idioms could be of great interest.

\section{Acknowledgments}

We thank Jaroslav Fowkes, Sharon Goldwater and Mirella Lapata for insightful comments and suggestions. We thank Johannes Dorn, Andreas Seve, and Marcel Bruch for help in integrating idioms into Snipmatch. This work was supported by Microsoft Research through its PhD Scholarship Programme. Charles Sutton was supported by the Engineering and Physical Sciences Research Council [grant number EP/K024043/1]. 


\section{References}

[1] M. Acharya, T. Xie, J. Pei, and J. Xu. Mining API patterns as partial orders from source code: from usage scenarios to specifications. In Joint Meeting of the European Software Engineering Conference and the ACM SIGSOFT Symposium on the Foundations of Software Engineering (ESEC/FSE), pages 25-34. ACM, 2007.

[2] M. Allamanis and C. Sutton. Mining source code repositories at massive scale using language modeling. In Working Conference on Mining Software Repositories (MSR), 2013.

[3] M. Allamanis, E. T. Barr, C. Bird, and C. Sutton. Learning natural coding conventions. In Symposium on the Foundations of Software Engineering (FSE), 2014.

[4] A. Bacchelli. Mining challenge 2013: StackOverflow. In Working Conference on Mining Software Repositories (MSR), 2013.

[5] B. S. Baker. A program for identifying duplicated code. Computing Science and Statistics, pages 49-49, 1993.

[6] H. A. Basit and S. Jarzabek. A data mining approach for detecting higher-level clones in software. IEEE Transactions on Software Engineering, 35(4):497-514, 2009.

[7] I. D. Baxter, A. Yahin, L. Moura, M. Sant'Anna, and L. Bier. Clone detection using abstract syntax trees. In International Conference on Software Maintenance, pages 368-377. IEEE, 1998.

[8] J. Campbell, A. Hindle, and J. N. Amaral. Syntax errors just aren't natural: Improving error reporting with language models. In Working Conference on Mining Software Repositories (MSR), 2014

[9] S. Chuan. JavaScript Patterns Collection. http://shichuan. github.io/javascript-patterns/, 2014. Visited Feb 2014.

[10] T. Cohn, P. Blunsom, and S. Goldwater. Inducing treesubstitution grammars. Journal of Machine Learning Research, 11:3053-3096, Nov 2010

[11] R. Cottrell, R. J. Walker, and J. Denzinger. Semi-automating small-scale source code reuse via structural correspondence. In Symposium on Foundations of Software Engineering (FSE), pages 214-225. ACM, 2008.

[12] Eclipse-Contributors. Eclipse JDT. eclipse.org/jdt, 2014. Visited Mar 2014.

[13] R. Falke, P. Frenzel, and R. Koschke. Empirical evaluation of clone detection using syntax suffix trees. Empirical Software Engineering, 13(6):601-643, 2008.

[14] M. Gabel and Z. Su. A study of the uniqueness of source code. In Symposium on Foundations of Software Engineering (FSE), pages 147-156. ACM, 2010.

[15] A. Gelman, J. B. Carlin, H. S. Stern, D. B. Dunson, A. Vehtari, and D. B. Rubin. Bayesian data analysis. CRC Press, 2013.

[16] S. J. Gershman and D. M. Blei. A tutorial on Bayesian nonparametric models. Journal of Mathematical Psychology, 56 (1):1-12, 2012.
[17] I. Grigorik. GitHub Archive. Www.githubarchive.org, 2014. Visited Mar 2014.

[18] A. Hindle, E. T. Barr, Z. Su, M. Gabel, and P. Devanbu. On the naturalness of software. In International Conference on Software Engineering (ICSE), 2012.

[19] N. L. Hjort. Bayesian Nonparametrics. Number 28. Cambridge University Press, 2010.

[20] R. Holmes, R. J. Walker, and G. C. Murphy. Approximate structural context matching: An approach to recommend relevant examples. IEEE Transactions on Software Engineering, 32(12):952-970, 2006.

[21] F. Jacob and R. Tairas. Code template inference using language models. In Annual Southeast Regional Conference, page 104. ACM, 2010.

[22] Java Idioms Editors. Java Idioms. http://c2 . com/ppr/ wiki/JavaIdioms/JavaIdioms.html, 2014. Visited Feb 2014.

[23] JetBrains. High-speed coding with Custom Live Templates. bit.1y/1o8R8Do, 2014. Visited Mar 2014.

[24] L. Jiang, G. Misherghi, Z. Su, and S. Glondu. Deckard: Scalable and accurate tree-based detection of code clones. In International Conference on Software Engineering (ICSE), pages 96-105. IEEE Computer Society, 2007.

[25] A. Jiménez, F. Berzal, and J.-C. Cubero. Frequent tree pattern mining: A survey. Intelligent Data Analysis, 14(6):603-622, 012010 .

[26] T. Kamiya, S. Kusumoto, and K. Inoue. CCFinder: a multilinguistic token-based code clone detection system for large scale source code. IEEE Transactions on Software Engineering, 28 (7):654-670, 2002.

[27] C. J. Kapser and M. W. Godfrey. "Cloning considered harmful" considered harmful: patterns of cloning in software. Empirical Software Engineering, 13(6):645-692, 2008.

[28] M. Kim, V. Sazawal, D. Notkin, and G. Murphy. An empirical study of code clone genealogies. In ACM SIGSOFT Software Engineering Notes, volume 30, pages 187-196. ACM, 2005.

[29] K. A. Kontogiannis, R. DeMori, E. Merlo, M. Galler, and M. Bernstein. Pattern matching for clone and concept detection. In Reverse Engineering, pages 77-108. Springer, 1996.

[30] R. Koschke, R. Falke, and P. Frenzel. Clone detection using abstract syntax suffix trees. In Working Conference on Reverse Engineering (WCRE), pages 253-262. IEEE, 2006.

[31] I. Kuzborskij. Large-scale pattern mining of computer program source code. Master's thesis, University of Edinburgh, 2011.

[32] Z. Li, S. Lu, S. Myagmar, and Y. Zhou. CP-Miner: Finding copy-paste and related bugs in large-scale software code. IEEE Transactions on Software Engineering, 32(3):176-192, 2006.

[33] P. Liang, M. I. Jordan, and D. Klein. Type-based MCMC. In Human Language Technologies: Annual Conference of the North American Chapter of the Association for Computational Linguistics (HLT/NAACL), pages 573-581, 2010. 
[34] C. J. Maddison and D. Tarlow. Structured generative models of natural source code. arXiv preprint arXiv:1401.0514, 2014.

[35] A. Menon, O. Tamuz, S. Gulwani, B. Lampson, and A. Kalai. A machine learning framework for programming by example. In International Conference on Machine Learning (ICML), pages 187-195, 2013.

[36] Microsoft Research. High-speed coding with Custom Live Templates. research. microsoft . com/apps/video/dl . aspx?id=208961, 2014. Visited Mar 2014.

[37] K. P. Murphy. Machine Learning: A Probabilistic Perspective. MIT Press, 2012.

[38] T. T. Nguyen, H. A. Nguyen, N. H. Pham, J. M. Al-Kofahi, and T. N. Nguyen. Graph-based mining of multiple object usage patterns. In Joint Meeting of the European Software Engineering Conference and the ACM SIGSOFT Symposium on the Foundations of Software Engineering (ESEC/FSE), pages 383-392. ACM, 2009.

[39] T. T. Nguyen, A. T. Nguyen, H. A. Nguyen, and T. N. Nguyen. A statistical semantic language model for source code. In Joint Meeting of the European Software Engineering Conference and the ACM SIGSOFT Symposium on the Foundations of Software Engineering (ESEC/FSE), 2013.

[40] Oracle. Java SE Documentation: Catching Multiple Exception Types and Rethrowing Exceptions with Improved Type Checking. http://docs.oracle.com/javase/7/docs/ technotes/guides/language/catch-multiple.html, 2014. Visited Feb 2014.

[41] P. Orbanz and Y. W. Teh. Bayesian nonparametric models. In Encyclopedia of Machine Learning. Springer, 2010.

[42] M. Post and D. Gildea. Bayesian learning of a tree substitution grammar. In Proceedings of the Association for Computational Linguistics (ACL), pages 45-48, 2009.

[43] E. Recommenders-Contributors. Eclipse SnipMatch. wiki.eclipse.org/Recommenders/Snipmatch, 2014. Visited Mar 2014.

[44] C. K. Roy and J. R. Cordy. A survey on software clone detection research. Technical report, Queen's University at Kingston, Ontario, 2007.

[45] C. K. Roy, J. R. Cordy, and R. Koschke. Comparison and evaluation of code clone detection techniques and tools: A qualitative approach. Science of Computer Programming, 74 (7):470-495, 2009.

[46] J. Sethuraman. A constructive definition of Dirichlet priors. Technical report, DTIC Document, 1991.

[47] Y. W. Teh. A hierarchical Bayesian language model based on Pitman-Yor processes. In Annual Meeting of the Association for Computational Linguistics (ACL), pages 985-992, 2006.

[48] Y. W. Teh and M. I. Jordan. Hierarchical Bayesian nonparametric models with applications. In N. Hjort, C. Holmes, P. Müller, and S. Walker, editors, Bayesian Nonparametrics: Principles and Practice. Cambridge University Press, 2010.
[49] A. Termier, M.-C. Rousset, and M. Sebag. Treefinder: a first step towards XML data mining. In International Conference on Data Mining (ICDM), pages 450-457. IEEE, 2002.

[50] R. Waldron. Principles of Writing Consistent, Idiomatic JavaScript. https://github.com/rwaldron/idiomatic. js/, 2014. Visited Feb 2014.

[51] J. Wang, Y. Dang, H. Zhang, K. Chen, T. Xie, and D. Zhang. Mining succinct and high-coverage API usage patterns from source code. In Working Conference on Mining Software Repositories (MSR), pages 319-328. IEEE, 2013.

[52] Wikibooks. More C++ Idioms. http://en.wikibooks. org/wiki/More_C\%2B\%2B_Idioms, 2013. Visited Feb 2014.

[53] C. K. Williams and C. E. Rasmussen. Gaussian Processes for Machine Learning, 2006.

[54] M. J. Zaki. Efficiently mining frequent trees in a forest. In Conference on Knowledge Discovery and Data Mining (KDD), pages 71-80. ACM, 2002.

[55] M. J. Zaki. Efficiently mining frequent trees in a forest: Algorithms and applications. IEEE Transactions on Knowledge and Data Engineering, 17(8):1021-1035, 2005.

[56] H. Zhong, T. Xie, L. Zhang, J. Pei, and H. Mei. MAPO: Mining and recommending API usage patterns. In European Conference on Object-Oriented Programming (ECOOP), pages 318-343. Springer, 2009. 